\title{
Method for controlling the shift invariance of optical correlators
}

\author{
Michael Levene, Gregory J. Steckman, and Demetri Psaltis
}

\begin{abstract}
Holographic correlators can implement many correlations in parallel. For most systems shift invariance limits the number of correlation templates that can be stored in one correlator. This is because the output plane must be divided among the individual templates in the system. When the system is completely shift invariant, the correlation peak from one correlator can shift into an area that has been reserved for a different template; in this case a shifted version of one object might be mistaken for a well-centered version of a different object. We describe a technique for controlling the shift invariance of a correlator system by moving the holographic material away from the Fourier plane. (C) 1999 Optical Society of America
\end{abstract}

OCIS code: $\quad 070.4550$.

\section{Introduction}

Pattern-recognition systems built from large numbers of correlation templates can be a powerful tool for many applications, including security, navigation, and human-computer interfaces. ${ }^{1}$ The robustness and the effectiveness of such systems improves if a larger number of templates is used. If templates are entered sequentially into the correlator, as the number of templates increases, the system speed decreases. However, holographic correlators are capable of performing multiple correlations simultaneously and displaying the results on the same detector at the output. We can distinguish the responses from different templates by dividing the output plane into individual domains, one for each template. If an input image shifts too much the corresponding correlation peak will move into a domain designated for another template, leading to a false identification. Limiting the shift invariance of the system prevents this error from occurring. The less shift invariance a system has, the smaller the indi-

When this research was performed, M. Levene was with Computation and Neural Systems and G. J. Steckman (steckman@ caltech.edu) and D. Psaltis were with the Department of Electrical Engineering, California Institute of Technology, Pasadena, California 91125. M. Levene is now with the Department of Applied and Engineering Physics, Cornell University, Ithaca, New York 14853.

Received 27 April 1998; revised manuscript received 15 September 1998.

0003-6935/99/020394-05\$15.00/0

(C) 1999 Optical Society of America vidual domains can be, and therefore more templates can be used in parallel. However, some amount of shift invariance is necessary for robust recognition. The trade-off between shift invariance and the number of templates used is an important design parameter for pattern-recognition systems.

The use of volume holograms is one method through which the shift invariance can be reduced. . $^{2,3}$ In this case Bragg selectivity can cause the correlation peak to disappear for shifts of the input image. Bragg selectivity in the plane defined by the reference and the signal beams is much greater than that for the out-of-plane direction, leading to an asymmetric correlation domain. This asymmetrism is a problem because most applications require symmetric correlation domains. The relatively weak control over the size of the domain in the out-of-plane direction also prevents us from storing more templates in this direction. In this paper we present a new method ${ }^{4}$ for controlling the shift invariance by shifting the hologram away from the Fourier plane and into the Fresnel region.

\section{Theory}

When the input image shifts in the optical correlator the plane-wave components at the Fourier plane all experience the same phase shift. This property results in shift invariance for thin holographic correlators stored in the Fourier plane. If the hologram is recorded away from the Fourier plane, however, the phase shift is not uniform across all plane-wave components. As a result, the various components of a shifted input image begin to add destructively, and 


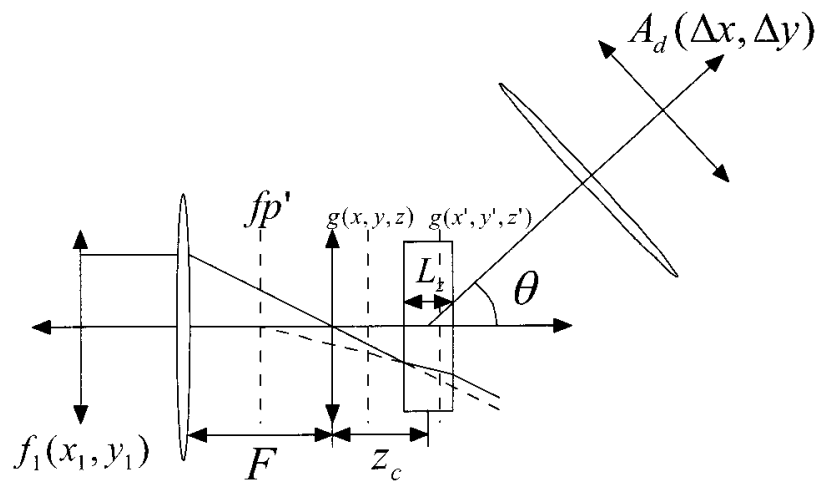

Fig. 1. Basic holographic correlator.

the correlation peak eventually disappears. The further the holographic material is from the Fourier plane, the greater the phase difference between the various component plane waves, and the more shift invariance is reduced.

Figure 1 shows a basic correlator system with the holographic material shifted a distance $z_{c}$ from the Fourier plane. A transparency $f_{1}\left(x_{1}, y_{1}\right)$, illuminated by monochromatic light of wavelength $\lambda$, produces a disturbance $g(x, y, z)$ in the Fresnel zone given (within the paraxial approximation and under the assumption that $z_{c}$ is small relative to $F$ ) by

$$
\begin{aligned}
g(x, y, z)= & \int_{-\infty}^{\infty} \int_{-\infty}^{\infty} f_{1}\left(x_{1}, y_{1}\right) \exp \left[-j \frac{k}{F}\left(x_{1} x+y_{1} y\right)\right] \\
& \times \exp \left[j \frac{k}{2 F^{2}}\left(x_{1}^{2}+y_{1}^{2}\right) z\right] \mathrm{d} x_{1} \mathrm{~d} y_{1}
\end{aligned}
$$

where $k=2 \pi / \lambda, F$ is the focal length of the lens, and $z$ is the distance from the Fourier plane. The refractive index, however, makes the Fourier plane appear to be shifted from its actual position. The field inside the material is then

$$
\begin{aligned}
g\left(x^{\prime}, y^{\prime}, z^{\prime}\right)= & \int_{-\infty}^{\infty} \int_{-\infty}^{\infty} f_{1}\left(x_{1}, y_{1}\right) \exp \left[-j \frac{k}{F}\left(x_{1} x+y_{1} y\right)\right] \\
& \times \exp \left\{j \frac { k } { 2 n F ^ { 2 } } ( x _ { 1 } ^ { 2 } + y _ { 1 } ^ { 2 } ) \left[z^{\prime}+\left(z_{c}-L_{z} / 2\right)\right.\right. \\
& \times(n-1)]\} \mathrm{d} x_{1} \mathrm{~d} y_{1}
\end{aligned}
$$

where $z_{c}$ is the distance from the Fourier plane to the center of the recording material.

A plane-wave reference incident at an angle $\theta$ interacts with the signal beam to record a hologram, as given by

$$
\left|\exp \left[j k\left(x^{\prime} \sin \theta^{\prime}+z^{\prime} \cos \theta^{\prime}\right)\right]+g\left(x^{\prime}, y^{\prime}, z^{\prime}\right)\right|^{2},
$$

where $\theta^{\prime}$ is the angle of the reference beam inside the medium.

Illuminating the hologram with a new input image $f_{2}\left(x_{2}, y_{2}\right)$ produces the diffracted field amplitude $A_{d}$

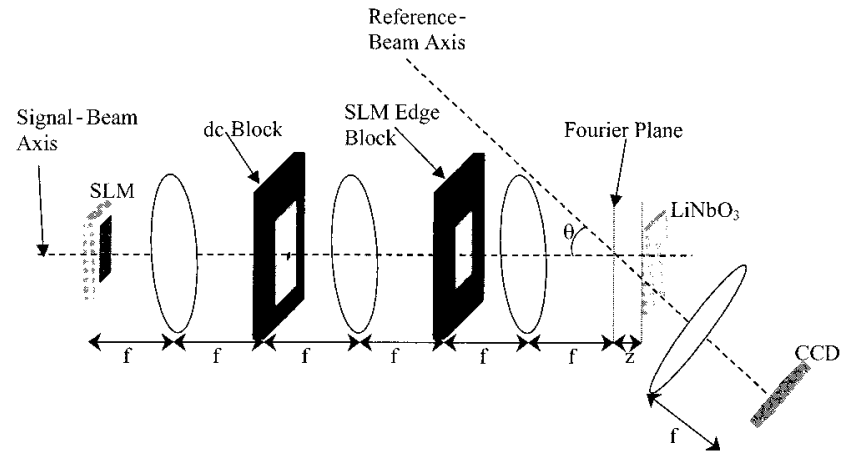

Fig. 2. Experimental correlator setup.

along the wave vector $\mathbf{k}_{d}$ given (within the Born approximation $^{5}$ ) by

$$
\begin{aligned}
A_{d}\left(\mathbf{k}_{d}\right)= & \iint \mathrm{d} x_{1} \mathrm{~d} y_{1} \iint \mathrm{d} x_{2} \mathrm{~d} y_{2} f_{1}\left(x_{1}, y_{1}\right) f_{2}\left(x_{2}, y_{2}\right) \\
& \times \iiint \exp \left\{-j \frac{k}{2 n F^{2}}\left(x_{1}^{2}-x_{2}^{2}+y_{1}^{2}-y_{2}^{2}\right)\right. \\
& \left.\times\left[z^{\prime}+\left(z_{c}-L_{z} / 2\right)(n-1)\right]\right\} \\
& \times \exp \left[j \frac{k}{F}\left(x_{1}-x_{2}\right) x^{\prime}\right] \\
& \times \exp \left[j \frac{k}{F}\left(y_{1}-y_{2}\right) y^{\prime}\right] \\
& \times \exp \left[j n\left(k \sin \theta^{\prime}-k_{d x}\right) x^{\prime}\right] \exp \left(-j n k_{d y} y^{\prime}\right) \\
& \times \exp \left\{j n ( k \operatorname { c o s } \theta ^ { \prime } - k _ { d z } ) \left[z^{\prime}+\left(z_{c}-L_{z} / 2\right)\right.\right. \\
& \times(n-1)]\} \mathrm{d} x^{\prime} \mathrm{d} y^{\prime} \mathrm{d} z^{\prime},
\end{aligned}
$$

where $k_{d x}, k_{d y}$, and $k_{d z}$ are the $x, y$, and $z$ components, respectively, of the diffracted wave vector such that $\left|\mathbf{k}_{d}\right|^{2}=k_{d x}{ }^{2}+k_{d y}{ }^{2}+k_{d z}{ }^{2}$.

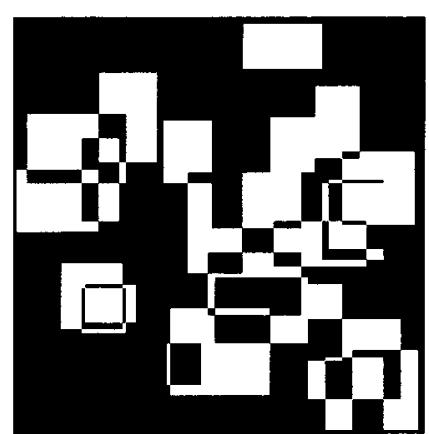

Fig. 3. Randomly generated image $120 \times 120$ pixels in size. 


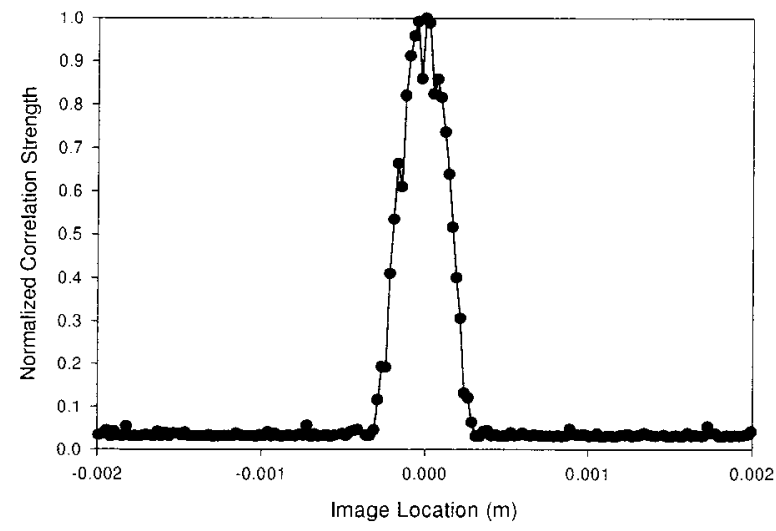

(a)

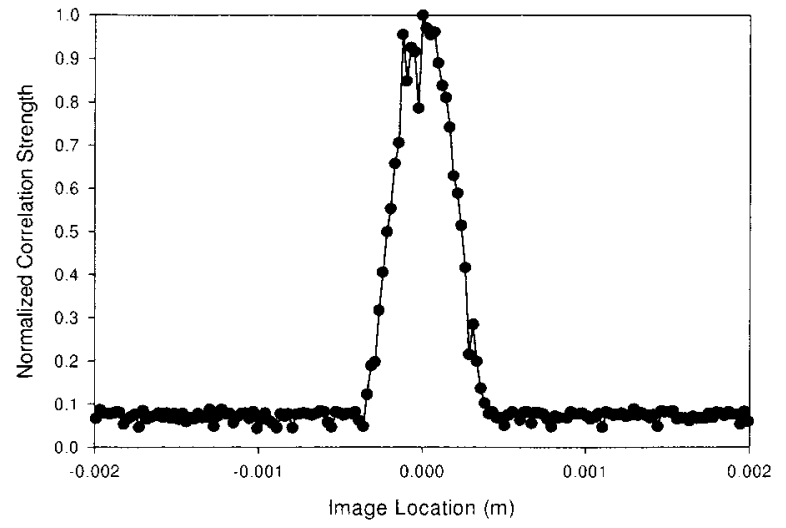

(b)

Fig. 4. Correlation strength versus image displacement for (a) in-plane and (b) out-of-plane shifts.

Performing the integral over the volume of the material with the change of variables $\tilde{z}=z^{\prime}-z_{c}$ yields

$$
\begin{aligned}
A_{d}\left(\mathbf{k}_{d}\right)= & \iint \mathrm{d} x_{1} \mathrm{~d} y_{1} \iint \mathrm{d} x_{2} \mathrm{~d} y_{2} f_{1}\left(x_{1}, y_{1}\right) f_{2}\left(x_{2}, y_{2}\right) \\
& \times \exp \left\{-j \frac{k}{2 n F^{2}}\left(x_{1}^{2}-x_{2}^{2}+y_{1}^{2}-y_{2}^{2}\right)\right. \\
& \left.\times\left[z_{c}+\left(z_{c}-L_{z} / 2\right)(n-1)\right]\right\} \exp \left\{j n \left(k \cos \theta^{\prime}\right.\right. \\
& \left.\left.-k_{d z}\right)\left[z_{c}+\left(z_{c}-L_{z} / 2\right)(n-1)\right]\right\} \\
& \times \operatorname{sinc}\left\{\frac { L _ { z } } { 2 \pi } \left[\frac{k}{2 n F^{2}}\left(x_{1}^{2}-x_{2}^{2}+y_{1}^{2}-y_{2}^{2}\right)\right.\right. \\
& \left.\left.+k n \cos \theta^{\prime}-n k_{d z}\right]\right\} \\
& \times \operatorname{sinc}\left\{\frac{L_{x}}{2 \pi}\left[\frac{k}{F}\left(x_{1}-x_{2}\right)+k n \sin \theta^{\prime}-n k_{d x}\right]\right\} \\
& \times \operatorname{sinc}\left\{\frac{L_{y}}{2 \pi}\left[\frac{k}{F}\left(y_{1}-y_{2}\right)-n k_{d y}\right]\right\},
\end{aligned}
$$

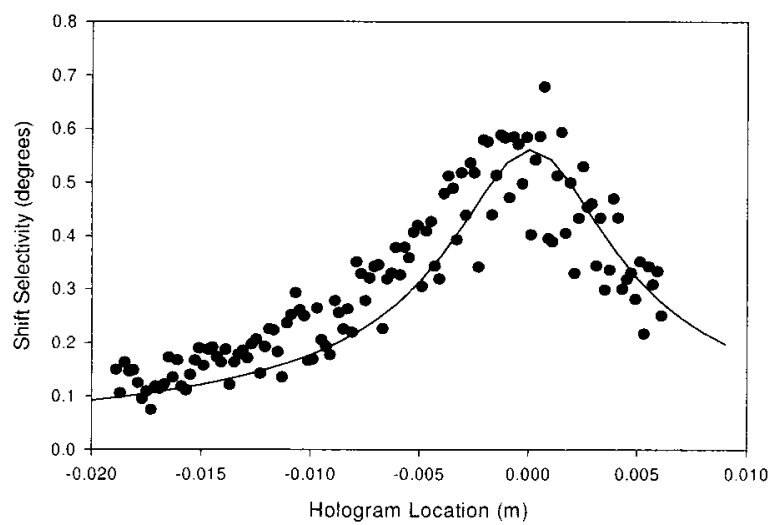

(a) with $\operatorname{sinc}(x)=(\sin \pi x) / \pi x$. The transverse dimensions $L_{x}$ and $L_{y}$ are large enough to approximate the last two sinc functions as delta functions. Integrating over $x_{2}$ and $y_{2}$ yields

$$
\begin{aligned}
A_{d}(\Delta x, \Delta y)= & \iint f_{1}\left(x_{1}, y_{1}\right) f_{2}\left(x_{1}+\Delta x, y_{1}+\Delta y\right) \\
& \times \exp \left\{j\left[z_{c}+\left(z_{c}-L_{c} / 2\right)(n-1)\right] \alpha\right\} \\
& \times \operatorname{sinc}\left(\frac{L_{z}}{2 \pi} \alpha\right) \mathrm{d} x_{1} \mathrm{~d} y_{1},
\end{aligned}
$$

where

$$
\begin{aligned}
\alpha= & \frac{k}{2 n F^{2}}\left[x_{1}^{2}-\left(x_{1}+\Delta x\right)^{2}+y_{1}^{2}-\left(y_{1}+\Delta y\right)^{2}\right] \\
& +k n \cos \theta^{\prime}-n k_{d z}, \\
\Delta x= & n F\left(\sin \theta^{\prime}-k_{d x} / k\right), \quad \Delta y=-n F k_{d y} / k .
\end{aligned}
$$

Equation (6) is the cross correlation between $f_{1}$ and $f_{2}$, with a sinc term from the Bragg selectivity and an exponential term involving the position of the hologram, $z_{c}$. Both the exponential and the sinc terms act as a window function on the correlation, attenu-

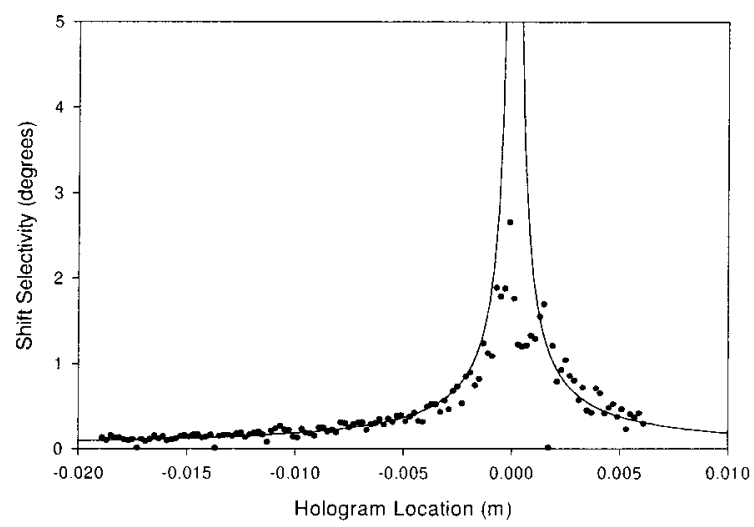

(b)

Fig. 5. (a) In-plane and (b) out-of-plane shift selectivity with the recording material displaced from the Fourier plane. 


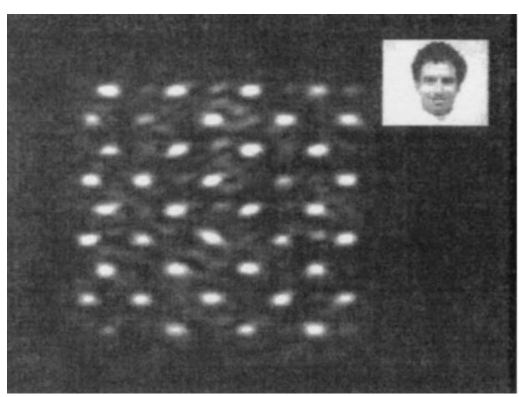

(a)

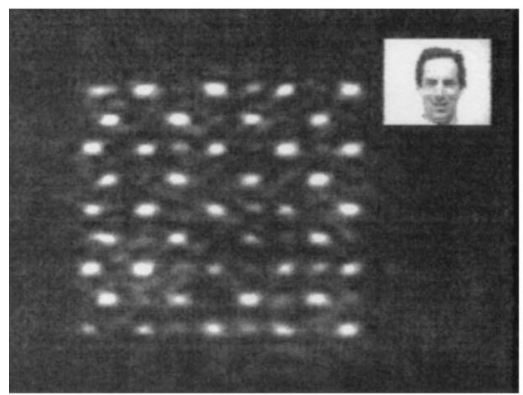

(b)

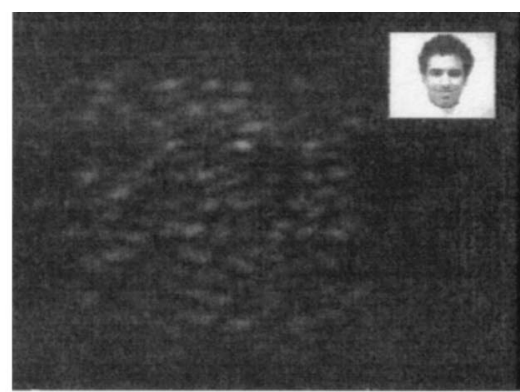

(c)

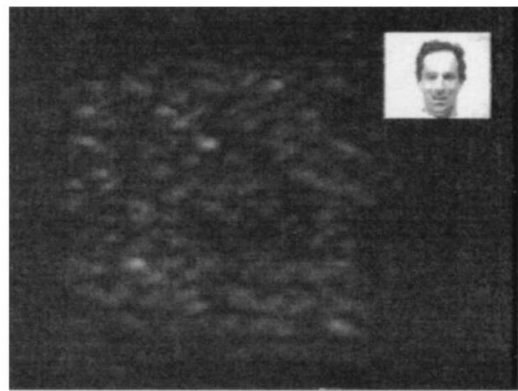

(d)

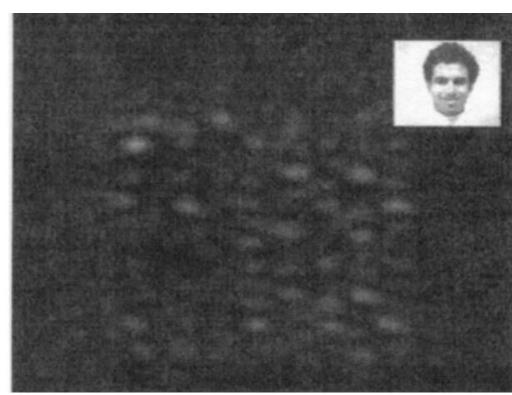

(e)

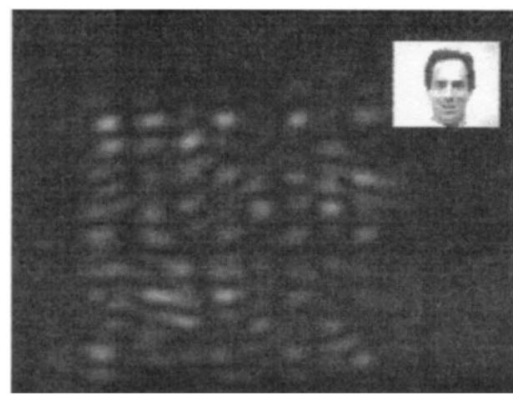

(f)

Fig. 6. Output of an array of correlators recorded $1 \mathrm{~cm}$ from the Fourier plane: (a), (b) centered input images; (c), (d) shifted input images; (e), (f) images recorded at the Fourier plane.

ating the signal for nonzero values of $\Delta x$ and $\Delta y$, i.e., for deviations from the center of the correlation domain. For volume holograms recorded at the Fourier plane, the exponential term becomes equal to one, and only the sinc term acts to limit shift invariance. Likewise, for thin holograms recorded away from the Fourier plane, the sinc term becomes negligible, and the exponential term becomes the limiting factor. The presence of this window function within the integral also acts to sharpen the correlation peaks by suppression of the sidelobes, as they occur at nonzero values of $\Delta x$ and $\Delta y$ even when the input is centered. Issues concerning shift invariance aside, the sidelobes of the correlations place an upper limit on how tightly the correlations can be packed in a conventional correlator. Attempting to store correlators together too closely results in the sidelobes from one template interfering with the neighboring template, reducing both outputs to noise.

\section{Experiment}

The experimental setup for the correlator that we used is shown in Fig. 2. An image of random white and black rectangles, shown in Fig. 3, was displayed on a portion of the liquid-crystal spatial light modulator (SLM), which has a resolution of $640 \times 480$ pixels and a 24- $\mu \mathrm{m}$ pixel pitch. A dc block in the Fourier plane of the first lens edge enhances the image before correlation. The filter behind the second lens blocks the edges of the SLM that were created by the edge-enhancement process of the dc block. If not blocked, the SLM creates an undesirable constant dc offset to the strength of the correlation, regardless of what image is presented on the SLM. The holographic material (a $250-\mu \mathrm{m}$-thick $\mathrm{LiNbO}_{3}$ crystal) is mounted on a motorized translation stage to enable computerized control of the location of the crystal relative to the Fourier plane. The signal beam is coincident with and the reference beam at a $25^{\circ}$ angle to the recording-material surface normal. A lens is placed along the path of the reference beam, and in its back focal plane a CCD camera is used to capture the intensity and the position of the correlation peak.

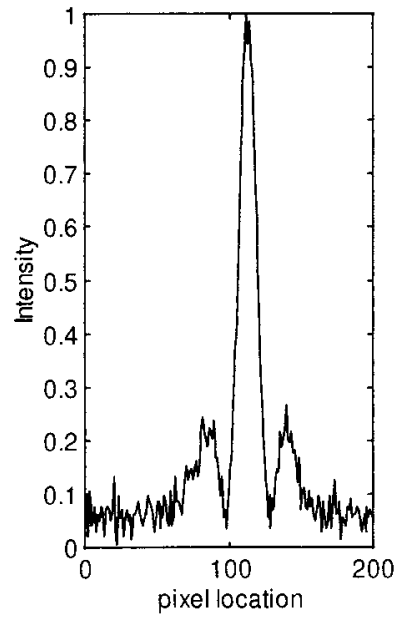

(a)

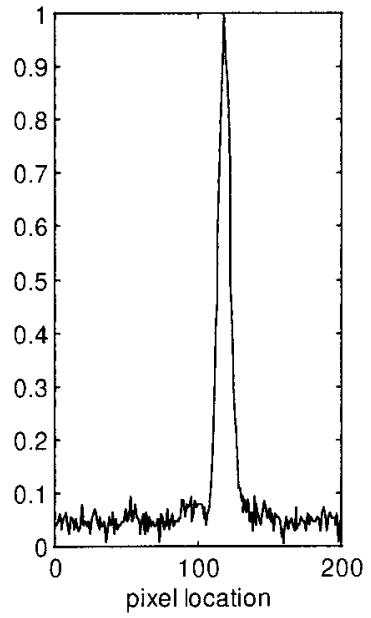

(b)
Fig. 7. Autocorrelation cross sections for (a) Fourier plane and (b) Fresnel zone recordings. 
The video signal from the CCD camera is digitized and analyzed by computer.

For each hologram displacement distance $z_{c}$ a hologram of the input pattern centered on the SLM is recorded. After recording, the reference beam is turned off, and the image on the SLM is correlated with the stored hologram. The input image is shifted electronically on the SLM. The image is first shifted horizontally (the in-plane direction) while it is centered vertically. For each horizontal location the intensity of the correlation peak and its location on the CCD are measured. The image is then shifted vertically (the out-of-plane direction) while it is centered horizontally; again, the peak intensity and the position are measured. The correlation measurements are taken under weak illumination to prevent both saturation of the CCD and erasure of the hologram.

Figure 4 shows typical curves of the peak intensity versus the image location for both horizontal and vertical displacements. The shift selectivity is measured as the width of such curves when they attain half of their maximum values. Plots of the shift selectivity for both the in-plane and the out-of-plane directions, together with the theoretical predictions, are shown in Fig. 5 as functions of the recordingmaterial location relative to the Fourier plane. The correlation integral derived in Section 2 was computed by use of a Monte Carlo technique with experimental values for the beam angle of $\left(25^{\circ}\right)$, the material thickness of $250 \mu \mathrm{m}$, and the index of refraction of 2.24 .

The experimental results agree well with the theoretical calculations over a large range of material displacements. Theory and experiment deviate most for out-of-plane shifts close to and at the Fourier plane, where the predicted value of the shift invariance shoots up to $25^{\circ}$. Figure 5 does not contain the full vertical range of the theoretical curve so that details of the wings could be evident.

Figures 6(a) and 6(b) show the output from an array of 81 correlators stored in $250-\mu \mathrm{m} \mathrm{LiNbO}_{3}$ that is displaced by $1 \mathrm{~cm}$ in front of the Fourier plane. Only two different faces were used, in an alternating fashion, as templates so that the overall correlation pattern could be monitored easily. In this experiment, the central reference-beam angle was $50^{\circ}$, and each reference beam was separated by $0.08^{\circ}$. Figures 6(c) and $6(\mathrm{~d})$ show the output when the input images are shifted just enough that their correlation peaks fall in the area reserved for the neighboring template; the peaks have disappeared, as intended, because of the position of the hologram in the Fresnel zone. Figures 6(e) and 6(f) show the output that results when the holograms are stored in the Fourier plane. In this case, the Bragg selectivity is not enough to prevent the sidelobes from interfering with neighboring templates, and the output of the system is noisy even for well-centered input images. This outcome shows that, by use of the Fresnel correlator system, more correlation templates can be stored and viewed unambiguously than would be possible in the Fourier plane. Figure 7 shows cross sections of autocorrelations for both the Fresnel and the Fourier plane holograms. The sidelobes of the Fresnel hologram are clearly suppressed relative to those for the Fourier hologram.

\section{Conclusion}

Although it is possible to rely on Bragg selectivity alone to control the shift invariance of holographic correlator systems, simply recording the holograms in the Fresnel zone allows for convenient control without the need to order material of the precise thickness necessary for a given application.

The authors thank the TRW Foundation and the National Science Foundation's (NSF) Engineering Research Center for providing support for this study. M. Levene acknowledges support from a NSF graduate traineeship.

\section{References}

1. B. Javidi and P. Réfrégier, eds., Optoelectronic Information Processing, Vol. PM54 of the SPIE Monographs and Handbooks Series (SPIE, Bellingham, Wash., 1997).

2. C. Gu, J. Hong, and S. Campbell, "2-D shift-invariant volume holographic correlator," Opt. Commun. 88, 309-314 (1992).

3. J. Yu, F. Mok, and D. Psaltis, "Capacity of optical correlators," in Spatial Light Modulators and Applications II, U. Efron, ed., Proc. SPIE 825, 128-135 (1987).

4. D. Psaltis and M. Levene, "Optical neural networks," in Vol. CR55 of SPIE Critical Review Series (SPIE, Bellingham, Wash., 1994), pp. 141-149.

5. J. D. Jackson, Classical Electrodynamics (Wiley, New York, 1975). 